\title{
Turning on the Left Side Electrode Changed Depressive State to Manic State in a Parkinson's Disease Patient Who Received Bilateral Subthalamic Nucleus Deep Brain Stimulation: A Case Report
}

\author{
Makoto Kinoshita ${ }^{1}$, Masahito Nakataki ${ }^{1}$, Ryoma Morigaki ${ }^{2,3,6}$, Satsuki Sumitani ${ }^{1,4}$, Satoshi Goto ${ }^{2,6}$, Ryuji Kaji ${ }^{5,6}$, \\ Tetsuro Ohmori ${ }^{1}$ \\ Departments of ${ }^{1}$ Psychiatry, ${ }^{2}$ Neurodegenerative Disorders Research, ${ }^{3}$ Neurosurgery, ${ }^{4}$ Support for Students with Special Needs, and ${ }^{5}$ Clinical \\ Neuroscience, Institute of Biomedical Sciences, Tokushima University Graduate School, ${ }^{6}$ Parkinson's Disease and Dystonia Research Center, \\ Tokushima University Hospital, Tokushima University, Tokushima, Japan
}

\begin{abstract}
No previous reports have described a case in which deep brain stimulation elicited an acute mood swing from a depressive to manic state simply by switching one side of the bilateral deep brain stimulation electrode on and off. The patient was a 68-year-old woman with a 10-year history of Parkinson's disease. She underwent bilateral subthalamic deep brain stimulation surgery. After undergoing surgery, the patient exhibited hyperthymia. She was scheduled for admission. On the first day of admission, it was clear that resting tremors in the right limbs had relapsed and her hyperthymia had reverted to depression. It was discovered that the left-side electrode of the deep brain stimulation device was found to be accidentally turned off. As soon as the electrode was turned on, motor impairment improved and her mood switched from depression to mania. The authors speculate that the lateral balance of stimulation plays an important role in mood regulation. The current report provides an intriguing insight into possible mechanisms of mood swing in mood disorders.
\end{abstract}

KEY WORDS: Subthalamic nucleus deep brain stimulation; Parkinson's disease; Manic episode; Depressive episode; Lateral balance.

\section{INTRODUCTION}

Parkinson's disease $(\mathrm{PD})$ is a progressive neurodegenerative disorder that manifests as resting tremors, muscular rigidity, bradykinesia, and postural instability. The etiology of PD is related to a lack of dopamine resulting from nigrostriatal neurodegeneration. Aside from pharmacotherapy as first-line treatment, stereotactic surgery is performed in patients whose motor symptoms are insufficiently improved by medication. ${ }^{1)}$

Although subthalamic nucleus deep brain stimulation (STN-DBS) is an effective operative treatment for PD, ${ }^{2)}$ the

Received: January 10, 2017 / Revised: February 24, 2017 Accepted: March 20, 2017

Address for correspondence: Makoto Kinoshita, MD, PhD Department of Psychiatry, Institute of Biomedical Sciences, Tokushima University Graduate School, 3-18-15, Kuramoto, Tokushima 770-8503, Japan

Tel: +81-88-633-7130, Fax: +81-88-633-7131

E-mail: knst5511mkt@sunny.ocn.ne.jp frequency of adverse psychiatric events is higher than in other methods of DBS. Meta-analyses have reported that following STN-DBS, depressive mood and manic state occur in $2 \%$ to $8 \%$, and $0.9 \%$ to $4 \%$ of patients, respectively. ${ }^{3,4)}$ However, it remains unclear what properties of the brain are related to mood changes after STN-DBS. Some studies have reported that lateralization in brain function is related to mood changes. Shimoda and Robinson ${ }^{5)}$ reported brain laterality in patients who exhibited poststroke depression. They showed that stroke in the left side of the brain induced depression more than stroke in the right side. Although poststroke manic symptoms are rare, patients who exhibited a poststroke manic state had more lesions in the right side of the brain than the left. ${ }^{6)}$ We report a case involving a patient whose depressive state switched to a manic state when the left-side electrode of the STN-DBS device was turned on.

(c) This is an Open-Access article distributed under the terms of the Creative Commons Attribution Non-Commercial License (http://creativecommons.org/licenses/by-nc/4.0) which permits unrestricted non-commercial use, distribution, and reproduction in any medium, provided the original work is properly cited. 


\section{CASE}

The patient was a 68-year-old woman with a 10-year history of PD, and no history of psychotic disease. Resting tremors in her left upper extremity was the first symptom. As symptoms worsened, muscular rigidity became apparent. Entacapone and pramipexole augmentation with L-dopa resulted in visual hallucinations. The Hoehn and Yahr $(\mathrm{H} \& \mathrm{Y})$ stage was 2 in the medication-on state and 4 in the medication-off state. The Unified Parkinson's Disease Rating Scale (UPDRS) Part III (motor section) scored 20 in the medication-on state and 33 in the medication-off state. She underwent bilateral STN-DBS surgery. After the surgery, the H\&Y stage was 2 and the UPDRS Part III score was 6. Although motor impairments improved with surgery, her visual hallucinations became worse and hyperthymia developed. She was referred to us for treatment of psychiatric symptoms. One year had passed since she underwent the STN-DBS procedure.

The patient was talkative and showed agitation. She was treated with olanzapine (maximum dose, $15 \mathrm{mg} /$ day) for three months. Because she did not improve, she was scheduled for admission to the Tokushima University Hospital (Tokushima, Japan). The final settings of STN-DBS were case positive and contact 2 and 3 negative, amplitude $2.5 \mathrm{~V}$, pulse width $60 \mu \mathrm{s}$, and frequency $130 \mathrm{~Hz}$. The therapy currents were $29 \mu \mathrm{A}$ (left-side) and $35 \mu \mathrm{A}$ (right-side). Ten days later, on the first day of admission, it was clear that her symptoms had changed. Resting tremors in the right limbs had relapsed and her hyperthymia had reverted to depression. Her volition was decreased and her voice was quiet. The Hamilton Depression Rating Scale (HAMD-17) score was 19, the Young Mania Rating Scale (YMRS) score was 0. Although she has not been diagnosed dementia before the admission, the Hasegawa Dementia Scale-Revised (HDS-R) score, consisting of 9 simple questions with a maximum score of 30 , slightly fell to 19 . No remarkable change was observed in the blood examination. Diffuse brain atrophy for her age was pointed out by the head computed topography (CT) scan. An adverse reaction to olanzapine was suspected, and the dose was reduced to $2.5 \mathrm{mg} /$ day. As her psychiatric symptoms showed no changes, we consulted the neurologist. It was discovered that the left-side electrode of the DBS device was accidentally turned off. As soon as the electrode was turned on, motor impair- ment improved and her mood switched from depression to mania. She became happy and was talkative again. The HAMD-17 score improved to 9, while the YMRS score was 18. The HDS-R score showed improvement to 22. Treatment with olanzapine (20 mg/day) and lithium carbonate (600 mg/day) was restarted. However, her symptoms showed only slight improvement (lithium concentration, $0.38 \mathrm{mEq} / \mathrm{L}$ ). Three months after admission, she was discharged from the hospital and moved to another health care facility.

\section{DISCUSSION}

The patient in the present case exhibited depressive mood only when the left-side electrode of the DBS device was turned off. As soon as it was turned on, she exhibited a manic state. Based on the blood examination, the head CT and the clinical history, we ruled out other possible cause of such a rapid mood switching. Tapered olanzapine did not improve her depressive state as well as did not develop manic state. Then we conclude that the left-side stimulation was crucial cause of her mood switch. No previous report has described a case in which a patient exhibited an acute mood switch from the depressive to manic state simply by turning one side of the bilateral electrode of the DBS device on and off. The STN exhibits anatomically localized function: the ventromedial side of the STN communicates with the limbic system, which regulates emotion. ${ }^{7)}$ Ulla et al. ${ }^{8)}$ recruited five PD patients who received bilateral STN-DBS, and altered the stimulation parameters. One patient exhibited a manic state when the deepest contact of the left-side electrode was stimulated. They also conducted a positron emission tomography study with these patients, which showed increased activity in the anterior cingulate cortex and right prefrontal cortex. Similarly, the patient in our case exhibited a manic state after the left-side electrode of the DBS device was turned on and, conversely, exhibited a depressive mood when the left-side electrode was turned off and the right side was on. In 24 PD patients who received bilateral STN-DBS, Campbell et al. ${ }^{9)}$ assessed mood changes under four settings (bilateral off; left unilateral stimulation only; right unilateral stimulation only; and bilateral stimulation), and demonstrated that the left-side electrode contributed to greater mood improvement than the right side. It is speculated that the left-side 
electrode stimulates the dorsal side of the STN, which affects parts of the limbic system involved in mood regulation. The lateral balance of stimulation is also believed to play an important role in mood regulation. The current report may suggest one possible mechanism of mood switches in mood disorders.

\section{REFERENCES}

1. Giugni JC, Okun MS. Treatment of advanced Parkinson's disease. Curr Opin Neurol 2014;27:450-460.

2. Kleiner-Fisman G, Herzog J, Fisman DN, Tamma F, Lyons KE, Pahwa R, et al. Subthalamic nucleus deep brain stimulation: summary and meta-analysis of outcomes. Mov Disord 2006;21 Supp/ 14:S290-S304.

3. Temel Y, Kessels A, Tan S, Topdag A, Boon P, VisserVandewalle V. Behavioural changes after bilateral subthalamic stimulation in advanced Parkinson disease: a systematic review. Parkinsonism Relat Disord 2006; 12:265-272.

4. Appleby BS, Duggan PS, Regenberg A, Rabins PV. Psychiatric and neuropsychiatric adverse events associated with deep brain stimulation: a meta-analysis of ten years' experience. Mov Disord 2007;22:1722-1728.

5. Shimoda K, Robinson RG. The relationship between poststroke depression and lesion location in long-term follow-up. Biol Psychiatry 1999;45:187-192.

6. Carota A, Bogousslavsky J. Mood disorders after stroke. Front Neurol Neurosci 2012;30:70-74.

7. Mallet L, Schüpbach M, N Diaye K, Remy P, Bardinet E, Czernecki $\mathrm{V}$, et al. Stimulation of subterritories of the subthalamic nucleus reveals its role in the integration of the emotional and motor aspects of behavior. Proc Natl Acad Sci USA 2007; 104:10661-10666.

8. Ulla M, Thobois S, Llorca PM, Derost P, Lemaire JJ, Chereau-Boudet I, et al. Contact dependent reproducible hypomania induced by deep brain stimulation in Parkinson's disease: clinical, anatomical and functional imaging study. J Neurol Neurosurg Psychiatry 2011;82:607-614.

9. Campbell MC, Black KJ, Weaver PM, Lugar HM, Videen TO, Tabbal SD, et al. Mood response to deep brain stimulation of the subthalamic nucleus in Parkinson's disease. J Neuropsychiatry Clin Neurosci 2012;24:28-36. 